\title{
Integers Powers of Certain Asymmetric Matrices
}

\author{
Roman Wituła and Damian Słota \\ Institute of Mathematics \\ Silesian University of Technology \\ Kaszubska 23, 44-100 Gliwice, Poland \\ \{roman.witula, damian.slota\}@polsl.pl
}

\begin{abstract}
In this paper we derive the recurrent formulae for any integers powers of certain asymmetric matrices five order which covers also constans tridiagonal matrices and some asymmetric (and symmetric) pentadiagonal matrices. Since we find also the Binet's formulae for the elements of the powers of these matrices, it is possible to operate with powers series of these matrices. We note that this method is alternative to the Jordan method decomposition.
\end{abstract}

Keywords: tridiagonal matrices, asymmetric matrices.

\section{Introduction}

The scope of the paper is to determine the recurrent formulae for the powers of certain asymmetric matrices belonging to the class $M_{5 \times 5}(\mathbb{C})$, involving constans tridiagonal matrices that have a very wide application. The recurrence method, which is an alternative to the Jordan's decomposition method, Leonard's algorithm, Putzer's algorithm and the method of characteristic polynomial 1 1234567 ], seems fast and facilitating the definition of integers powers of any matrices. The paper is a follow up on [8] (which is inspired by [910], see also [11]), where the cases of the third and fourth order asymmetric matrices are discussed and where also the complex powers of these matrices are defined. Apart from the proposed formulae, the authors also present a computational example of the application of this procedure.

\section{Basic Recurrence Formulae}

Let us set now:

$$
\mathbf{G}_{5}(a, b, c, d, e, f, g, A, B, y):=\left[\begin{array}{ccccc}
a & y b & y^{2} c & y^{3} e & y^{4} g \\
b & A & y d & y^{2} f & y^{3} e \\
c & d & B & y d & y^{2} c \\
e & f & d & A & y b \\
g & e & c & b & a
\end{array}\right]
$$

for any $a, b, c, d, e, f, g, A, B, y, \in \mathbb{C}$.

G. Allen et al. (Eds.): ICCS 2009, Part I, LNCS 5544, pp. 580-587, 2009.

(C) Springer-Verlag Berlin Heidelberg 2009 
Lemma 1. Let $a_{1}, b_{1}, c_{1}, d_{1}, e_{1}, f_{1}, g_{1}, A_{1}, B_{1}, y, \in \mathbb{C}$. If

$$
\begin{aligned}
d_{1} & =b_{1}+y e_{1}, \\
f_{1} & =c_{1}+y g_{1}, \\
A_{1} & =a_{1}+y c_{1}, \\
B_{1} & =a_{1}+y c_{1}+y^{2} g_{1},
\end{aligned}
$$

then we get

$$
\begin{aligned}
& \left(\mathbf{G}_{5}\left(a_{1}, b_{1}, c_{1}, d_{1}, e_{1}, f_{1}, g_{1}, A_{1}, B_{1}, y\right)\right)^{k}= \\
& \quad=\mathbf{G}_{5}\left(a_{k}, b_{k}, c_{k}, d_{k}, e_{k}, f_{k}, g_{k}, A_{k}, B_{k}, y\right)
\end{aligned}
$$

for every $k \in \mathbb{N}$, where the following recurrence formulae hold:

$$
\begin{aligned}
a_{k+1} & =a_{1} a_{k}+y b_{1} b_{k}+y^{2} c_{1} c_{k}+y^{3} e_{1} e_{k}+y^{4} g_{1} g_{k}, \\
b_{k+1} & =b_{1} a_{k}+A_{1} b_{k}+y d_{1} c_{k}+y^{2} f_{1} e_{k}+y^{3} e_{1} g_{k}, \\
c_{k+1} & =c_{1} a_{k}+B_{1} c_{k}+y^{2} c_{1} g_{k}+d_{1} d_{k} \\
& =B_{1} c_{k}+B_{k} c_{1}-y c_{1} c_{k}+d_{1} d_{k}, \\
e_{k+1} & =e_{1} a_{k}+f_{1} b_{k}+d_{1} c_{k}+A_{1} e_{k}+y b_{1} g_{k}, \\
g_{k+1} & =g_{1} a_{k}+e_{1} b_{k}+c_{1} c_{k}+b_{1} e_{k}+a_{1} g_{k}, \\
d_{k} & =b_{k}+y e_{k}, \\
f_{k} & =c_{k}+y g_{k}, \\
A_{k} & =a_{k}+y c_{k}, \\
B_{k} & =a_{k}+y c_{k}+y^{2} g_{k} .
\end{aligned}
$$

We note that if additionally we have $c_{1}=g_{1}=e_{1}=0$ then $G_{5}\left(a_{1}, \ldots, y\right)$ is a tridiagonal matrix.

The proof of the lemma by an easy induction arguments follows.

Now we want to find the Binet's formulae for the elements $a_{k}, b_{k}, c_{k}, e_{k}$ and $g_{k}$. To this aim it is sufficient to designate twenty five coefficients $\xi_{i}$ for $\xi \in\{\alpha, \beta, \gamma, \delta, \varepsilon\}$ and $i=1,2,3,4,5$, for which the following system of equations hold:

$$
\begin{aligned}
a_{k} & =\alpha_{1} t^{k}+\beta_{1} u^{k}+\gamma_{1} v^{k}+\delta_{1} w^{k}+\varepsilon_{1} z^{k}, \\
y^{1 / 2} b_{k} & =\alpha_{2} t^{k}+\beta_{2} u^{k}+\gamma_{2} v^{k}+\delta_{2} w^{k}+\varepsilon_{2} z^{k}, \\
y c_{k} & =\alpha_{3} t^{k}+\beta_{3} u^{k}+\gamma_{3} v^{k}+\delta_{3} w^{k}+\varepsilon_{3} z^{k}, \\
y^{3 / 2} e_{k} & =\alpha_{4} t^{k}+\beta_{4} u^{k}+\gamma_{4} v^{k}+\delta_{4} w^{k}+\varepsilon_{4} z^{k} \\
y^{2} g_{k} & =\alpha_{5} t^{k}+\beta_{5} u^{k}+\gamma_{5} v^{k}+\delta_{5} w^{k}+\varepsilon_{5} z^{k},
\end{aligned}
$$

for every $k \in \mathbb{N}$ and for some $t, u, v, w, z \in \mathbb{C}$ independent on $k$. 
From identities (3)-(7) and (12)-(16) if we compare the coefficients of powers $u^{k+1}, v^{k+1}, w^{k+1}$ and $z^{k+1}$ it is easily established the following system of equations

$$
\begin{aligned}
& \xi_{1}=\xi_{1}^{2}+\xi_{2}^{2}+\xi_{3}^{2}+\xi_{4}^{2}+\xi_{5}^{2}, \\
& \xi_{2}=2 \xi_{1} \xi_{2}+2 \xi_{2} \xi_{3}+2 \xi_{3} \xi_{4}+2 \xi_{4} \xi_{5}, \\
& \xi_{3}=2 \xi_{1} \xi_{3}+2 \xi_{2} \xi_{4}+2 \xi_{3} \xi_{5}+\xi_{2}^{2}+\xi_{3}^{2}+\xi_{4}^{2}, \\
& \xi_{4}=2 \xi_{1} \xi_{4}+2 \xi_{2} \xi_{3}+2 \xi_{3} \xi_{4}+2 \xi_{2} \xi_{5}, \\
& \xi_{5}=2 \xi_{1} \xi_{5}+2 \xi_{2} \xi_{4}+\xi_{3}^{2},
\end{aligned}
$$

for every $\xi \in\{\alpha, \beta, \gamma, \delta, \varepsilon\}$.

Sketch of solution of the system (17)-(21):

Substracting equations: (21) from (17) and next (18) from (20) we obtain respectively:

$$
\begin{aligned}
& \xi_{1}-\xi_{5}=\left(\xi_{1}-\xi_{5}\right)^{2}+\left(\xi_{2}-\xi_{4}\right)^{2}, \\
& \xi_{2}-\xi_{4}=2 \xi_{1}\left(\xi_{2}-\xi_{4}\right)+2 \xi_{5}\left(\xi_{4}-\xi_{2}\right)=2\left(\xi_{2}-\xi_{4}\right)\left(\xi_{1}-\xi_{5}\right),
\end{aligned}
$$

i.e.,

$$
\left(\xi_{2}-\xi_{4}\right)\left(1-2\left(\xi_{1}-\xi_{5}\right)\right)=0 .
$$

From (23) we have, that either $\xi_{2}-\xi_{4}=0$ or $\xi_{1}-\xi_{5}=\frac{1}{2}$. If $\xi_{2}=\xi_{4}$ then by (22) either $\xi_{1}=\xi_{5}$ or $\xi_{1}-\xi_{5}=1$. On the other hand, if $\xi_{1}-\xi_{5}=\frac{1}{2}$, then by (22) $\xi_{2}-\xi_{4}= \pm \frac{1}{2}$. Finally, the equations system (17)-(21) is extended to include one of the following four equations:

1. $\xi_{2}=\xi_{4}$ and $\xi_{1}=\xi_{5}$,

2. $\xi_{2}=\xi_{4}$ and $\xi_{1}-\xi_{5}=1$,

3. $\xi_{1}-\xi_{5}=\frac{1}{2}$ and $\xi_{2}-\xi_{4}=\frac{1}{2}$,

4. $\xi_{1}-\xi_{5}=\frac{1}{2}$ and $\xi_{2}-\xi_{4}=-\frac{1}{2}$,

reducing the number of solutions to 28 vectors from $\mathbb{R}^{5}$ (all calculations were performed in Mathematica). Surely, out of the 28 vectors, we are interested in only five different ones. It should be indicated that in view of equations (3)-(7), the required vectors must comply with the following orthogonal conditions:

$$
\sum_{i=1}^{5} \zeta_{i} \eta_{i}=\sum_{i=1}^{5} \zeta_{i} \eta_{5-i}=0
$$

for every $\zeta, \eta \in\{\alpha, \beta, \gamma, \delta, \varepsilon\}, \zeta \neq \eta$. From this point, it is easy to derive the following solution:

$$
\left[\begin{array}{lllll}
\alpha_{1} & \beta_{1} & \gamma_{1} & \delta_{1} & \varepsilon_{1} \\
\alpha_{2} & \beta_{2} & \gamma_{2} & \delta_{2} & \varepsilon_{2} \\
\alpha_{3} & \beta_{3} & \gamma_{3} & \delta_{3} & \varepsilon_{3} \\
\alpha_{4} & \beta_{4} & \gamma_{4} & \delta_{4} & \varepsilon_{4} \\
\alpha_{5} & \beta_{5} & \gamma_{5} & \delta_{5} & \varepsilon_{5}
\end{array}\right]=\left[\begin{array}{ccccc}
\frac{1}{12} & \frac{1}{4} & \frac{1}{3} & \frac{1}{4} & \frac{1}{12} \\
\frac{-1}{4 \sqrt{3}} & \frac{1}{4} & 0 & -\frac{1}{4} & \frac{1}{4 \sqrt{3}} \\
\frac{1}{6} & 0 & -\frac{1}{3} & 0 & \frac{1}{6} \\
\frac{-1}{4 \sqrt{3}} & -\frac{1}{4} & 0 & \frac{1}{4} & \frac{1}{4 \sqrt{3}} \\
\frac{1}{12} & -\frac{1}{4} & \frac{1}{3} & -\frac{1}{4} & \frac{1}{12}
\end{array}\right]
$$


Moreover, we can generate the following formulae

$$
\begin{aligned}
t & =a_{1}-b_{1} \sqrt{3 y}+2 c_{1} y+g_{1} y^{2}-e_{1} \sqrt{3 y^{3}} \\
u & =a_{1}+b_{1} \sqrt{y}-g_{1} y^{2}-e_{1} \sqrt{y^{3}}, \\
v & =a_{1}-c_{1} y+g_{1} y^{2}, \\
w & =a_{1}-b_{1} \sqrt{y}-g_{1} y^{2}+e_{1} \sqrt{y^{3}}, \\
z & =a_{1}+b_{1} \sqrt{3 y}+2 c_{1} y+g_{1} y^{2}+e_{1} \sqrt{3 y^{3}} .
\end{aligned}
$$

Corollary 1. We have

$$
\left[\begin{array}{lllll}
a & c & 0 & 0 & 0 \\
b & a & c & 0 & 0 \\
0 & b & a & c & 0 \\
0 & 0 & b & a & c \\
0 & 0 & 0 & b & a
\end{array}\right]^{r}=\mathbf{G}_{5}\left(\alpha_{r}, \beta_{r}, \gamma_{r}, \delta_{r}, \varepsilon_{r}, f_{r}, g_{r}, A_{r}, B_{r}, \frac{c}{b}\right)
$$

where

$$
\begin{aligned}
\alpha_{r} & =\frac{1}{12} t^{r}+\frac{1}{4} u^{r}+\frac{1}{3} v^{r}+\frac{1}{4} w^{r}+\frac{1}{12} z^{r}, \\
y^{1 / 2} \beta_{r} & =\frac{-1}{4 \sqrt{3}} t^{r}+\frac{1}{4} u^{r}-\frac{1}{4} w^{r}+\frac{1}{4 \sqrt{3}} z^{r}, \\
y \gamma_{r} & =\frac{1}{6} t^{r}-\frac{1}{3} v^{r}+\frac{1}{6} z^{r}, \\
y^{3 / 2} \varepsilon_{r} & =\frac{-1}{4 \sqrt{3}} t^{r}-\frac{1}{4} u^{r}+\frac{1}{4} w^{r}+\frac{1}{4 \sqrt{3}} z^{r}, \\
y^{2} g_{r} & =\frac{1}{12} t^{r}-\frac{1}{4} u^{r}+\frac{1}{3} v^{r}-\frac{1}{4} w^{r}+\frac{1}{12} z^{r}, \\
y^{1 / 2} \delta_{r} & =y^{1 / 2} \beta_{r}+y^{3 / 2} \varepsilon_{r}=\frac{\sqrt{3}}{6}\left(z^{r}-t^{r}\right), \\
4 y f_{r} & =4\left(y \gamma_{r}+y^{2} g_{r}\right)=t^{r}-u^{r}-w^{r}+z^{r}, \\
4 A_{r} & =4\left(\alpha_{r}+y \gamma_{r}\right)=t^{r}+u^{r}+w^{r}+z^{r}, \\
3 B_{r} & =3\left(\alpha_{r}+y \gamma_{r}+y^{2} g_{r}\right)=t^{r}+v^{r}+z^{r},
\end{aligned}
$$

and

$$
\begin{aligned}
& t=a-\sqrt{3 b c}, \\
& u=a+\sqrt{b c}, \\
& v=a, \\
& w=a-\sqrt{b c}, \\
& z=a+\sqrt{3 b c} .
\end{aligned}
$$

Remark 1. All formulas (2)-(16) and (30)-(39) hold true also for all $k, r \in \mathbb{Z}$, since $\mathbf{G}_{5}\left(a_{1}, b_{1}, \ldots, g_{1}, A_{1}, B_{1}, y\right)$ is the diagonal matrix (see also [8] for more 
comments). The powers in the respective formulas should be chosen in the following way:

$$
x^{r}:=\exp (r(\ln |x|+i \operatorname{Arg} x)) .
$$

\section{Example}

Now the numerical example of application of our formulae will be given. Let us set: $a_{1}=1, b_{1}=1, c_{1}=-1, e_{1}=-1, g_{1}=1, d_{1}=1-i=\sqrt{2} e^{-i \pi / 4}$, $f_{1}=-1+i=\sqrt{2} e^{i 3 \pi / 4}, A_{1}=1-i=\sqrt{2} e^{-i \pi / 4}, B_{1}=-i, y=i$. For these values, we have

$$
\mathbf{G}_{5}\left(a_{1}, b_{1}, \ldots, B_{1}, y\right)=\left[\begin{array}{rrrrrr}
1 & i & 1 & i & 1 \\
1 & 1-i & 1+i & 1-i & i \\
-1 & 1-i & -i & 1+i & 1 \\
-1 & -1+i & 1-i & 1-i & i \\
1 & -1 & -1 & 1 & 1
\end{array}\right] .
$$

By (25)-29) we get

$$
\begin{aligned}
t & =1-\sqrt{3 i}-2 i-1+\sqrt{-3 i} \\
& =-\exp \left(\frac{1}{2}\left(\ln 3+i \frac{\pi}{2}\right)\right)-2 i+\exp \left(\frac{1}{2}\left(\ln 3+i \frac{3 \pi}{2}\right)\right) \\
& =-\sqrt{3} \exp \left(i \frac{\pi}{4}\right)-2 i+\sqrt{3} \exp \left(i \frac{3 \pi}{4}\right) \\
& =-\sqrt{6}-2 i \\
u & =2+i \sqrt{2} \\
v & =i \\
w & =2-i \sqrt{2} \\
z & =\sqrt{6}-2 i
\end{aligned}
$$

Then by (2) we have

$$
\mathbf{G}_{5}^{k}\left(a_{1}, b_{1}, \ldots, B_{1}, y\right)=\mathbf{G}_{5}\left(a_{k}, b_{k}, \ldots, B_{k}, y\right), \quad k \in \mathbb{Z},
$$

where

$$
\begin{aligned}
a_{k}= & \frac{1}{12}(-(\sqrt{6}+2 i))^{k}+\frac{1}{4}(2+i \sqrt{2})^{k}+\frac{1}{3} i^{k}+\frac{1}{4}(2-i \sqrt{2})^{k} \\
& +\frac{1}{12}(\sqrt{6}-2 i)^{k}, \\
e^{i \pi / 4} b_{k}= & -\frac{\sqrt{3}}{12}(-(\sqrt{6}+2 i))^{k}+\frac{1}{4}(2+i \sqrt{2})^{k}-\frac{1}{4}(2-i \sqrt{2})^{k} \\
& +\frac{\sqrt{3}}{12}(\sqrt{6}-2 i)^{k}, \\
i c_{k}= & \frac{1}{6}(-(\sqrt{6}+2 i))^{k}-\frac{1}{3} i^{k}+\frac{1}{6}(\sqrt{6}-2 i)^{k},
\end{aligned}
$$




$$
\begin{aligned}
e^{-i \pi / 4} e_{k}= & -\frac{\sqrt{3}}{12}(-(\sqrt{6}+2 i))^{k}-\frac{1}{4}(2+i \sqrt{2})^{k}+\frac{1}{4}(2-i \sqrt{2})^{k} \\
& +\frac{\sqrt{3}}{12}(\sqrt{6}-2 i)^{k}, \\
g_{k}= & -\frac{1}{12}(-(\sqrt{6}+2 i))^{k}+\frac{1}{4}(2+i \sqrt{2})^{k}-\frac{1}{3} i^{k}+\frac{1}{4}(2-i \sqrt{2})^{k} \\
& -\frac{1}{12}(\sqrt{6}-2 i)^{k}, \\
2 e^{i \pi / 4} d_{k}= & (2+i \sqrt{2})^{k}-(2-i \sqrt{2})^{k}, \\
4 i f_{k}= & (-(\sqrt{6}+2 i))^{k}-(2+i \sqrt{2})^{k}-(2-i \sqrt{2})^{k}+(\sqrt{6}-2 i)^{k}, \\
4 A_{k}= & (-(\sqrt{6}+2 i))^{k}+(2+i \sqrt{2})^{k}+(2-i \sqrt{2})^{k}+(\sqrt{6}-2 i)^{k}, \\
3 B_{k}= & (-(\sqrt{6}+2 i))^{k}+i^{k}+(\sqrt{6}-2 i)^{k} .
\end{aligned}
$$

For example, for $k=-1$ we get

$$
\mathbf{G}_{5}^{-1}\left(a_{1}, \ldots, B_{1}, y\right)=\left[\begin{array}{rrrrr}
\frac{1}{6}-\frac{3 i}{10} & \frac{2}{15}-\frac{i}{30} & -\frac{2}{5} & -\frac{2}{15}-\frac{i}{30} & \frac{1}{6}+\frac{3 i}{10} \\
-\frac{1}{30}-\frac{2 i}{15} & \frac{1}{6}+\frac{i}{10} & \frac{1}{10}+\frac{i}{10} & -\frac{1}{10}-\frac{i}{6} & -\frac{2}{15}-\frac{i}{30} \\
\frac{2}{5} & \frac{1}{10}-\frac{i}{10} & -\frac{i}{5} & \frac{1}{10}+\frac{i}{10} & -\frac{2}{5} \\
\frac{1}{30}-\frac{2 i}{15} & \frac{1}{10}+\frac{i}{6} & \frac{1}{10}-\frac{i}{10} & \frac{1}{6}+\frac{i}{10} & \frac{2}{15}-\frac{i}{30} \\
\frac{1}{6}+\frac{3 i}{10} & \frac{1}{30}-\frac{2 i}{15} & \frac{2}{5} & -\frac{1}{30}-\frac{2 i}{15} & \frac{1}{6}-\frac{3 i}{10}
\end{array}\right] .
$$

\section{Final Remarks}

$1^{\circ}$ It's some natural problem how to defined some power series of $\mathbf{G}_{5}$. For example, how $e^{\mathbf{G}_{5}}$ should be defined? By Lemma 1 it can be defined in the following way:

$$
\begin{gathered}
\exp \left(\mathbf{G}_{5}(a, b, c, d, e, f, g, A, B, y)\right) \approx \\
\approx \sum_{k=0}^{N} \frac{1}{k !} \mathbf{G}_{5}\left(a_{k}, b_{k}, c_{k}, d_{k}, e_{k}, f_{k}, g_{k}, A_{k}, B_{k}, y\right)= \\
\quad=\mathbf{G}_{5}\left(\sum_{k=0}^{N} \frac{1}{k !} a_{k}, \sum_{k=0}^{N} \frac{1}{k !} b_{k}, \ldots, \sum_{k=0}^{N} \frac{1}{k !} B_{k}, y\right),
\end{gathered}
$$

where $N$ can be chosen arbitrary with a given accuracy, because of the Binet's formulae (12)-(16). On the other hand, we have the following exact formula:

$$
\exp \left(\mathbf{G}_{5}(a, b, c, d, e, f, g, A, B, y)\right)=\mathbf{G}_{5}(\widehat{a}, \widehat{b}, \widehat{c}, \widehat{d}, \widehat{e}, \widehat{f}, \widehat{g}, \widehat{A}, \widehat{B}, y)
$$


where

$$
\left[\begin{array}{r}
\widehat{a} \\
y^{1 / 2} \widehat{b} \\
y \widehat{c} \\
y^{3 / 2} \widehat{e} \\
y^{2} \widehat{g}
\end{array}\right]=\left[\begin{array}{ccccc}
\frac{1}{12} & \frac{1}{4} & \frac{1}{3} & \frac{1}{4} & \frac{1}{12} \\
\frac{-1}{4 \sqrt{3}} & \frac{1}{4} & 0 & -\frac{1}{4} & \frac{1}{4 \sqrt{3}} \\
\frac{1}{6} & 0 & -\frac{1}{3} & 0 & \frac{1}{6} \\
\frac{-1}{4 \sqrt{3}} & -\frac{1}{4} & 0 & \frac{1}{4} & \frac{1}{4 \sqrt{3}} \\
\frac{1}{12} & -\frac{1}{4} & \frac{1}{3} & -\frac{1}{4} & \frac{1}{12}
\end{array}\right]\left[\begin{array}{c}
e^{t} \\
e^{u} \\
e^{v} \\
e^{w} \\
e^{z}
\end{array}\right]
$$

for the definition of the parameters $t, u, v, w, z$ see formulae (25)-(29), and

$$
\begin{aligned}
& \widehat{d}=\widehat{b}+y \widehat{e}, \\
& \widehat{f}=\widehat{c}+y \widehat{g}, \\
& \widehat{A}=\widehat{a}+y \widehat{c}, \\
& \widehat{B}=\widehat{a}+y \widehat{c}+y^{2} \widehat{g} .
\end{aligned}
$$

In the connection with these deliberations it is intriguing in some sense that Mathematica have some problem with symbolic Jordan decomposition of the matrix $\mathbf{G}_{5}\left(a_{1}, b_{1}, \ldots, B_{1}, y\right)$ from our example.

$2^{\circ}$ Why are only the matrices $5 \times 5$ discussed in the paper? It turned out that generalizations of the case $3 \times 3$ (from [8]) onto $4 \times 4$ and $5 \times 5$ matrices are unique in a certain sense. The case of the $6 \times 6$ matrix:

$$
\mathbf{G}_{6}:=\left[\begin{array}{cccccc}
a & y b & y^{2} c & y^{3} e & y^{4} g & y^{5} k \\
b & A & y d & y^{2} f & y^{3} h & y^{4} g \\
c & d & B & y d & y^{2} f & y^{3} e \\
e & f & d & B & y d & y^{2} c \\
g & h & f & d & A & y b \\
k & g & e & c & b & a
\end{array}\right]
$$

with respective recurrence relations for its elements don't work. In consequence the generalization $n \times n, n \geq 6$, are not so strong (it is necessary to use less number of parameters or others recurrence relations).

\section{References}

1. Abu-Saris, R., Ahmad, W.: Avoiding eigenvalues in computing matrix power. Amer. Math. Monthly 112, 450-454 (2005)

2. Barakat, R., Baumann, E.: $M$ th power on $N \times N$ matrix and its connection with the generalized Lucas polynomial. J. Math. Phys. 10, 1474-1476 (1969)

3. Beckenbach, E.F.: Modern Mathematics for the Engineer. McGraw-Hill, New York (1961)

4. Elaydi, S.N., Harris Jr., W.A.: On the computation of $A^{N}$. SIAM Rev. 40, 965-971 (1998)

5. Fulmer, E.P.: Computation of the matrix exponential. Amer. Math. Monthly 82, 156-159 (1975)

6. Leonard, I.E.: The matrix exponential. SIAM Rev. 38, 507-512 (1996) 
7. Putzer, E.J.: Avoiding the Jordan canonical form in the discussion of linear systems with constant coefficients. Amer. Math. Monthly 73, 2-7 (1966)

8. Wituła, R., Słota, D.: Some phenomenon of the powers of certain tridiagonal and asymmetric matrices. Appl. Math. Comput. 202, 348-359 (2008)

9. Samarskii, A.A., Gulin, A.V.: The Stability of the Difference Schemes. Nauka, Moscow (in Russian) (1973)

10. Wituła, R., Słota, D.: On computing the determinants and inverses of some special type of tridiagonal and constant-diagonals matrices. Appl. Math. Comput. 189, 514-527 (2007)

11. Kilic, E.: On a constant-diagonals matrix. Appl. Math. Comput. 204, 184-190 (2008) 